\title{
Cross sectional early mitral flow velocity profiles from colour Doppler
}

\author{
STEIN O SAMSTAD, HANS G TORP, * DAVID T LINKER, * OLE ROSSVOLL, \\ TERJE SKJAERPE, ERLING JOHANSEN, * KJELL KRISTOFFERSEN, * \\ BJOERN A J ANGELSEN, * LIV HATLE
}

From the Section of Cardiology, Department of Medicine, Regional Hospital, and Institute of Biomedical Engineering, University of Trondheim, Norway

SUMMARY Instantaneous cross sectional flow velocity profiles from early mitral flow in 10 healthy men were constructed by time interpolation of the velocity data from each point in sequentially delayed two dimensional digital Doppler ultrasound maps. This interpolation allows correction of the artificially produced skewness of velocities across the flow sector caused by the time taken to scan the flow sector for velocity recording of pulsatile blood flow. These results suggested that early mitral flow studied in an apical four chamber view is variably skewed both at the leaflet tips and at the annulus. The maximum flow velocity overestimated the cross sectional mean velocity at the same time by a factor of $1 \cdot 2-2 \cdot 2$. Also the maximum time velocity integral overestimated the cross sectional mean time velocity integral to the same extent.

This cross sectional skew must be taken into account when calculation of blood flow is based on recordings with pulsed wave Doppler ultrasound from a single sample volume.

Pulsed wave Doppler has been used to record the flow patterns in non-stenotic mitral valves. ${ }^{1-3}$ The method has an accurate time resolution, but the velocities are recorded from only a small area of the valve orifice. If the velocity varies across the orifice a recording from one sample volume may differ from the mean cross sectional velocity. Recently colour flow Doppler has been used to describe flow velocity patterns. ${ }^{46}$ This method has accurate spatial resolution, but the poor time resolution might introduce errors in the measurement of pulsatile flow because of the sweep time across the flow sector, which is needed to update the colour flow maps.

To overcome the limited spatial information given by pulsed wave Doppler and the poor time resolution of colour flow Doppler a new method was developed. Digital Doppler ultrasound two dimensional flow maps, obtained with the ultrasound beam directed in parallel to the blood velocities, were used to construct instantaneous flow velocity profiles across the mitral orifice.

The technique was used to examine mitral flow in

Requests for reprints to Dr Stein O Samstad, Section of Cardiology, Department of Medicine, Regional Hospital, University of Trondheim, N-7006 Trondheim, Norway.

Accepted for publication 28 February 1989 early diastole in healthy men. We examined these flow velocity profiles to estimate the error that may occur with pulsed wave Doppler when a limited sample volume is used to measure the mean velocity across the orifice.

\section{Patients and methods}

Ten healthy men, aged 12 to 42 years (mean 31 years), consented to the study. None had clinical, auscultatory, electrocardiographic, or echocardiographic evidence of heart disease.

The ultrasound measurements were made with a CFM 700 system (VingMed Sound). This is a combined cross sectional echocardiographic and two dimensional Doppler flow velocity system where the flow sector is constructed by colour coding the velocity information from 64 sample volumes along each of 64 sequentially transmitted ultrasound beams in each flow velocity map. The velocity in each sample volume is calculated by an autocorrelation function on the frequency and the intensity of the backscattered ultrasound signal. ${ }^{78}$ We used a $3 \mathrm{MHz}$ transducer with the high pass filter set at $21 \mathrm{~cm} / \mathrm{s}$, and by scrolling the baseline the upper velocity limit was $120 \mathrm{~cm} / \mathrm{s}$. The lateral resolution of the ultrasound beam, defined as a $50 \%$ decrease in 
backscattered signal from the centreline of the beam, was $2 \cdot 1-2.5 \mathrm{~mm}$ at a depth of $8 \mathrm{~cm}$ from the transducer when the flow sector angle was set to $30^{\circ}$.

Each ultrasound beam is incrementally delayed relative to the preceding beam because of the time needed to send and receive several pulses before a new beam can be sent in the next direction. The sweep time of each flow map with a flow sector angle of $30^{\circ}$ varies from 40 to $114 \mathrm{~ms}$ for different instrument set-ups and transducer. The actual sweep times are measured directly on the transducer controller inside the instrument. The flow sector can be moved by a track ball both laterally and radially in relation to the tissue sector. The width and the radial extension of the flow sector can also be adjusted while the $64 \times 64$ sampling volumes are maintained. The distances from the transducer to the areas of interest in the tissue and flow velocity maps are measured accurately by the analysis facilities of the instrument.

The men were examined in the left lateral recumbent position. The transducer was located at the apical window to give a four chamber view of the heart. Adjustment of the position and direction of the transducer and of the orientation of the flow sector reduced to a minimum the angle between the radial direction of the flow sector and the main direction of the mitral flow. The septal and lateral parts of the mitral annulus were visualised, and the sector plane was adjusted in the anterioposterior plane until optimal flow signals were obtained from the inflow channel. During data acquisition the patients were asked to stop breathing in passive end expiration with open airways.

Electrocardiographically triggered flow maps of the mitral area were made from sequential beats, and the start of the flow sweep relative to the $R$ peak was increasingly delayed with increments of $20 \mathrm{~ms}$ from one beat to the next. The increases in sweep delay were adjusted manually. The first sweep was recorded before the start of passive atrioventricular flow and the last sweep after onset of flow caused by left atrial systole. The number of sweeps ranged from 13 to 19 (mean (SD) $16 \cdot 1(2 \cdot 3)$ ) in the ten men. The serial time gated recording of these flow sweeps obtained from each man was used for analysis of the flow patterns both at the mitral annulus and at the leaflet tips. None of the recording periods exceeded 25 seconds.

The ultrasound recordings were transferred to an external computer as raw Doppler data from the front end of the CFM 700 in real time or as digital velocity data from the replay memory of the instrument (fig 1). In addition, the recordings were stored on videotape.

In subjects 1 to 4 , the raw colour Doppler data were transferred from the front end of the instrument

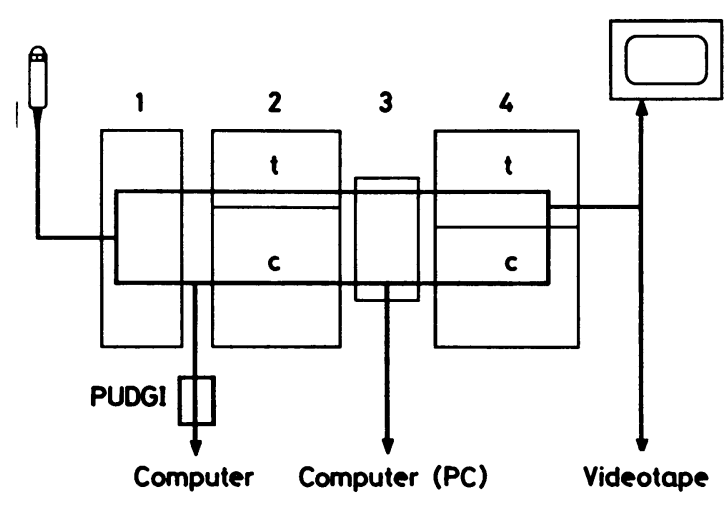

Fig 1 Ultrasound system. Solid line represents the signal passage from the transducer (upper left) to the final presentation as cross sectional tissue and colour flow map on the monitor of the instrument (upper right). Four major levels of signal processing in the instrument are:

(1) Instrument front end connected to the transducer. A custom made portable ultrasound data grabber and interface (PUDGI) capable of transferring the raw ultrasound data in real time to an external computer can be connected to the front end. The real time transfer of the ultrasound data enables post-processing of the raw digital Doppler flow data without its being influenced by the processing units of the instrument. (2) Data processing unit. The tissue data (t) and the Doppler colour flow data (c) are processed separately before the data are passed to the scan converter (4). (3) Digital replay memory with two megabyte storage capacity. The processed data on tissue and Doppler colour flow are passed to the replay memory of the instrument in parallel with the data transfer from the processing unit (2) to the scan converter (4). At this level the tissue and Doppler colour flow data are handled separately and the replay memory is continuously updated as new data are passed through the system, with a simultaneous erasing of the oldest data in the memory. In freeze mode of the instrument the content of the digital replay memory can be replayed from the monitor for measurements and tape recording. These data can also be transferred to an external computer for the analysis performed in this article. (4) Tissue and colour flow images are mixed in the scan converter for combined presentation on the monitor. The Doppler colour flow sector is overlayed on the tissue sector from the same transducer sweep cycle.

via a custom made ultrasound data grabber and interface (PUDGI) to a Stride 460 computer (Stride Micro, Reno, NV). The raw two dimensional Doppler data were processed by a computer program that $N$ emulated the velocity estimation in the ultrasound $Q$ instrument, and the velocity data were presented as a 0 colour flow map displayed on a monitor or as velocity

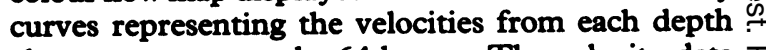
along an arc across the 64 beams. The velocity data were stored for further processing (fig 1).

The recordings from subjects $5-1.0$ were transferred from the replay memory of the ultrasound 
instrument to an external computer (IBM PC compatible). At this level of data processing the Doppler data are available as digital velocity information from each of the 64 times 64 points of the flow sector from each recorded map. The selected number of consecutively recorded maps from each patient could be transferred by custom software in the ultrasound instrument. The velocities along an arc of the 64 beams at defined depths from the recordings could also be transferred from these files to a standard data file for further processing.

These standard data files, containing information on the instrument set-up as well as the digital Doppler data, were loaded into a commercial spreadsheet program (Microsoft Excel, Microsoft Corporation) where the calculations needed to correct for the sweep time and generate undistorted velocity profiles were made.

In some of the recorded maps, velocity data from some points along the arc of the ultrasound beams were missing. These "holes" were regarded as artefacts introduced by the instrument. The missing values were corrected in the spreadsheet program by comparing the velocity data close to the "hole" and replacing the missing values with the average of the two neighbouring values from the same sweep before the time interpolation procedure.

Both transfer procedures give rapid collection of high quality flow velocity data unaffected by errors introduced by the display system, video recorder, or re-digitisation of a colour coded display.

Any of the 64 arcs across the flow sector can be used to generate velocity profiles. The depth from the transducer to the displayed cardiac structures of the tissue sector can be measured from the actual recording by the replay memory of the instrument.

Since the mitral flow is pulsatile, the sweep time from one side of the flow sector to the other distorts the velocity profiles constructed from arcs of sample volu nes across the flow sector. To compensate for this distortion, data obtained from sequentially delayed flow sweeps were interpolated. Each sweep of the ultrasound beam was triggered by the $R$ wave of the electrocardiogram. Consecutive sweeps were delayed by $20 \mathrm{~ms}$ in relation to the previous one (fig 2 $b$ and c). We excluded recordings where the $R R$ intervals of the various heart cycles deviated more than $20 \mathrm{~ms}$ from the median.

Thus the position and velocity information from each point along the selected arcs relative to the $R$ wave of the electrocardiogram were known for each flow sweep, and linear interpolation between the velocities from each of the sequentially delayed recordings was done. The interpolation varied across the arc to adjust for the sweep time and resulted in an estimate of the cross sectional flow profile at a single

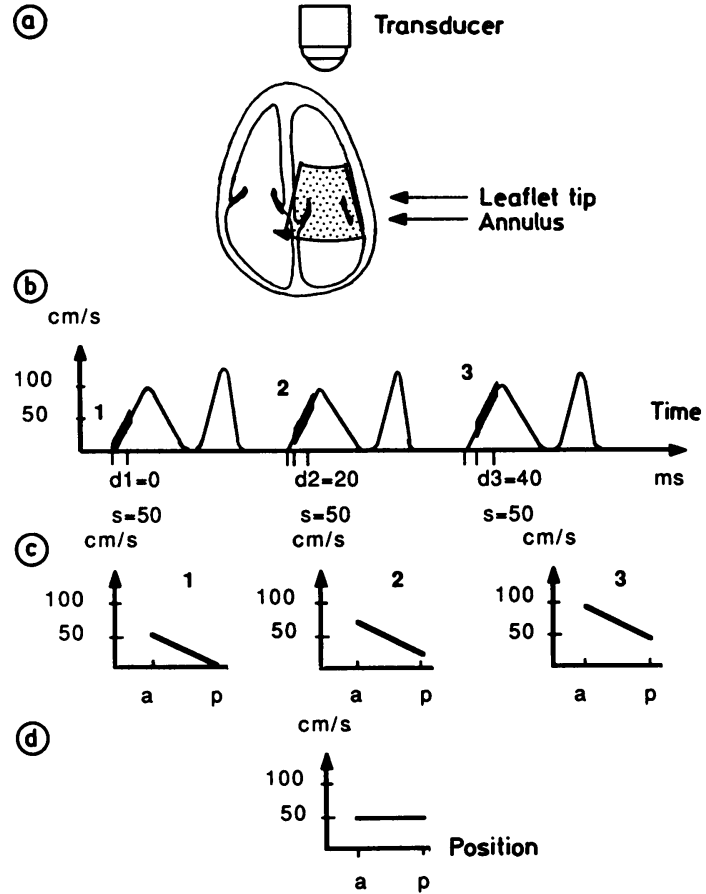

Fig 2 (a) Schematic drawing of the heart in apical four chamber view. The mitral flow velocity distribution in this example is assumed to be flat. The ultrasound Doppler two dimensional sector is $30^{\circ}$; the two dimensional Doppler ultrasound sweep direction is from right to left, sweep time = $50 \mathrm{~ms}$. Horizontal arrows indicate the depths of cross sectional flow profiles in this study. (b) Pulsed wave Doppler recording of the pulsatile flow in the mitral orifice at both levels with overlayed velocity changes during a recording period (s) of $50 \mathrm{~ms}$, and where the recording period is sequentially delayed (d) by $20 \mathrm{~ms}$ relative to the flow start from one flow period to the other; $d l=0 \mathrm{~ms}, d 2=20 \mathrm{~ms}$, and $d 3=40$ ms. (c) Velocity traces across the width of the mitral orifice in panel (a) as obtained when recorded with the ultrasound Doppler two dimensional system. Velocities are given along the $y$ axis and the position in the mitral orifice along the $x$ axis ( $a=$ anterior leaflet, $p=$ posterior leaflet). Numbers 1 to 3 represent sequentially delayed two dimensional sweeps of the flow pulses 1 to 3 in (b). The duration of each sweep is $50 \mathrm{~ms}$. Note the artificial skewing caused by the lag in data collection. (d) Instantaneous flow velocity distribution across the width of the orifice in panel (a). Velocities are given along the $y$ axis, and position in the mitral orifice along the $x$ axis ( $a=$ anterior leaflet, $p=$ posterior leaflet). This flow profile is calculated by linear time interpolation of the velocity traces 1 to 3 in panel (c), at 50 ms from start of flow.

point in time (fig 2d). Since the time lag of the updating of the colour flow map is compensated for by the calculation procedure, we called this calculated cross sectional flow velocity distribution the 
instantaneous flow velocity profile. An instantaneous flow velocity profile could be calculated for any time in the flow cycle and at any depth of the two dimensional Doppler flow velocity sector and the results displayed as a three dimensional plot for each depth (fig 3). We calculated the instantaneous flow velocity at the leaflet tips and at the mitral annulus in all $10 \mathrm{men}$, and measured the actual depths at the time of maximum separation of the mitral leaflets in early diastole.

\section{STATISTICAL ANALYSIS}

Results are given as ranges and sample means (SD).

\section{Results}

The results were plotted as velocity profiles versus time to give a three dimensional presentation of the
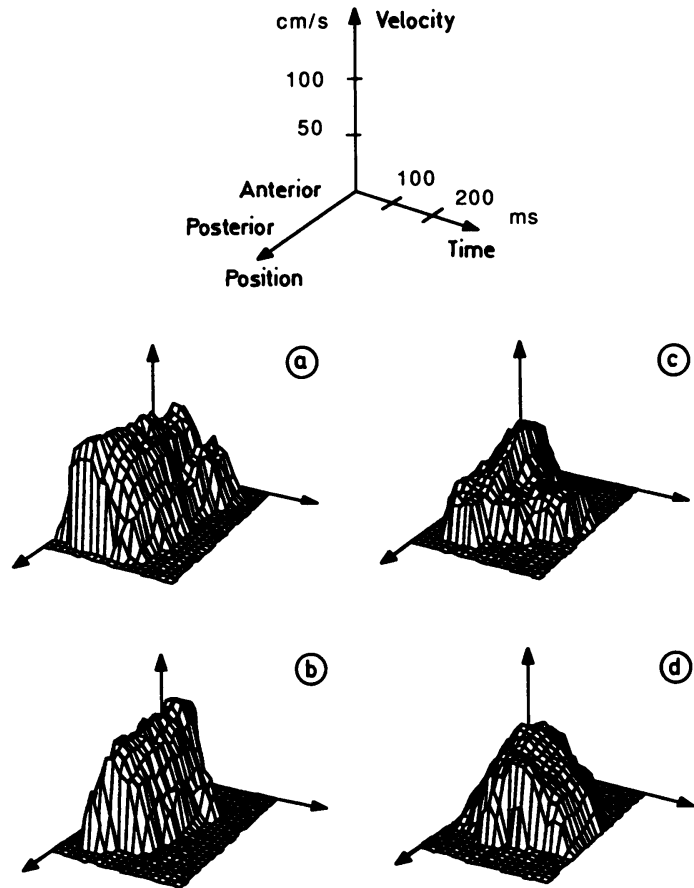

Fig 3 Plots of instantaneous early mitral flow velocity profiles against time. Axis scaling and orientation are shown at the top of the figure. Plots $a$ and $b$ are from subject number 2 , recorded from the level of the leaflet tips, $(a)$ and at the level of the mitral annulus (b). Plots (c) and (d) are from subject number 7 at the level of the leaflet tips and annulus respectively. In these two healthy men the instantaneous flow profiles at the leaflet tips are skewed, but the maximum velocities are at different positions in the valve orifice-in subject 2 at the posterior leaflet and in subject 7 at the anterior leaflet. The velocity profiles were skewed at the level of the mitral annulus in both men, but the highest velocities were recorded at the anterior leaflet. data. The profiles were to some extent skewed in all subjects, both at the annulus and at the tip of the mitral valve leaflets. The plots in fig 3 show different orientations of the skew in two patients. In patient 2 the skew is toward the posterior leaflet, while in patient 7 the skew is toward the anterior leaflet. At the level of the annulus the highest velocities were recorded anteriorly in both men.

The maximal instantaneous velocity versus the simultaneous cross sectional mean velocity gives an indication of the degree of skewness at the time of peak flow. The difference between maximum velocity and cross sectional mean velocity at the leaflet tips varied from $14 \mathrm{~cm} / \mathrm{s}$ in subject 2 up to $41 \mathrm{~cm} / \mathrm{s}$ in subject 6 (table). The mean differences for all subjects were $24.4 \mathrm{~cm} / \mathrm{s}$ and $23.1 \mathrm{~cm} / \mathrm{s}$ at the level of the leaflet tips and at the annulus respectively. Figure 4 shows the relative locations of the maximum velocity within the valve orifice and the maximum velocity as a proportion of the cross sectional mean velocity at the same time for each man at the levels of the leaflet tips and the annulus.

The time velocity integral is the integral of velocity over time for a specific point in the arc. This is the value used to estimate volume flow when recording with pulsed wave Doppler from a single sample
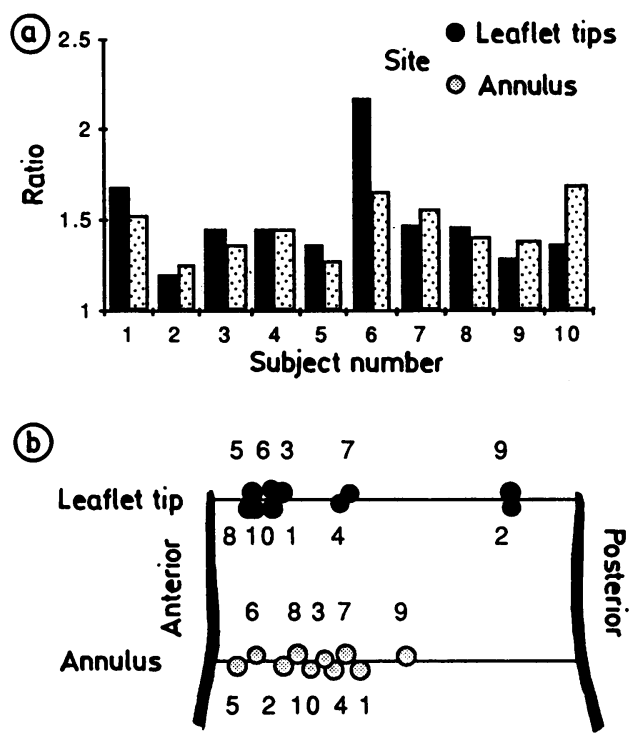

Fig 4 (a) Ratio of the maximum velocity to the mean cross sectional velocity at the time of maximum flow velocity of early mitral flow, at the leaflet tips (solid bars) and at the annulus (patterned bars). (b) Site of the maximum velocity within the mitral orifice is shown for each subject at the level of the tips (solid dots) and the annulus (open circles). Leaflet margins are indicated as solid vertical lines. 
Table Maximum flow velocity and time-velocity integral of early mitral inflow compared with the simultaneous mean velocity and mean time-velocity integral across the mitral orifice at the level of the leaflet tips and the annulus

\begin{tabular}{|c|c|c|c|c|c|c|c|c|c|c|}
\hline \multirow[b]{2}{*}{ No } & \multirow[b]{2}{*}{$\begin{array}{c}\text { Age } \\
(y r)\end{array}$} & \multirow[b]{2}{*}{$\begin{array}{l}\text { HR } \\
\text { (beats/min) }\end{array}$} & \multicolumn{4}{|c|}{ Velocity $(V)(\mathrm{cm} / \mathrm{s})$} & \multicolumn{4}{|c|}{ Time-velocity integral $(I)(\mathrm{cm})$} \\
\hline & & & $\begin{array}{l}\text { Tip } \\
\operatorname{Max} V\end{array}$ & Mean $V$ & $\begin{array}{l}\text { Annulus } \\
\operatorname{Max} V\end{array}$ & Mean $V$ & $\begin{array}{l}\text { Tip } \\
\operatorname{Max} I\end{array}$ & Mean I & $\begin{array}{l}\text { Annulus } \\
\text { Max I }\end{array}$ & Mean I \\
\hline $\begin{array}{c}1 \\
2 \\
3 \\
4 \\
5 \\
6 \\
7 \\
8 \\
9 \\
10 \\
\text { Mean } \\
\text { SD }\end{array}$ & $\begin{array}{r}38 \\
42 \\
32 \\
28 \\
42 \\
33 \\
28 \\
34 \\
25 \\
12 \\
31 \\
9\end{array}$ & $\begin{array}{l}55 \\
41 \\
76 \\
67 \\
60 \\
68 \\
56 \\
59 \\
58 \\
74 \\
61 \\
10\end{array}$ & $\begin{array}{l}59 \\
79 \\
70 \\
50 \\
80 \\
75 \\
55 \\
73 \\
80 \\
77 \\
69 \cdot 8 \\
11 \cdot 1\end{array}$ & $\begin{array}{l}36 \\
65 \\
33 \\
32 \\
57 \\
34 \\
37 \\
54 \\
60 \\
46 \\
45 \cdot 4 \\
12.6\end{array}$ & $\begin{array}{l}80 \\
82 \\
57 \\
55 \\
67 \\
66 \\
58 \\
77 \\
82 \\
74 \\
69 \cdot 8 \\
10.6\end{array}$ & $\begin{array}{l}57 \\
65 \\
34 \\
26 \\
51 \\
39 \\
37 \\
52 \\
66 \\
40 \\
46 \cdot 7 \\
13.5\end{array}$ & $\begin{array}{r}6 \cdot 2 \\
12.0 \\
6.8 \\
8 \cdot 1 \\
10 \cdot 3 \\
11 \cdot 3 \\
6 \cdot 8 \\
10 \cdot 1 \\
11 \cdot 1 \\
8.7 \\
9 \cdot 1 \\
2 \cdot 1\end{array}$ & $\begin{array}{r}3.7 \\
10.0 \\
4.7 \\
5.6 \\
7.6 \\
5.2 \\
4.6 \\
6.9 \\
8.6 \\
6.4 \\
6.3 \\
2.0\end{array}$ & $\begin{array}{r}7.5 \\
11.0 \\
5.6 \\
6.8 \\
7.7 \\
10.0 \\
7.5 \\
7.2 \\
8.9 \\
8.3 \\
8.1 \\
1.6\end{array}$ & $\begin{array}{l}4.9 \\
8.7 \\
4 \cdot 1 \\
4 \cdot 7 \\
6 \cdot 0 \\
6 \cdot 0 \\
4 \cdot 8 \\
5 \cdot 1 \\
6 \cdot 4 \\
4 \cdot 9 \\
5 \cdot 6 \\
1 \cdot 3\end{array}$ \\
\hline
\end{tabular}

Tip, measurements at the level of mitral leaflet tips; annulus, measurements at the level of the mitral annulus; HR, heart rate; Max V, maximum velocity of the early mitral flow; Mean V, cross sectional mean velocity at the time of maximum velocity at the defined level; Max I, time velocity integral from the point in the flow sector at the defined level giving maximum value of early mitral flow; Mean I, mean cross sectional time velocity integral of early mitral flow at defined depth in the flow sector.

volume. The maximum time velocity integral is the maximum found for a given arc. The mean time velocity integral is the arithmetic mean of the integral values across this arc.

The difference between the maximum and the simultaneous cross sectional mean time velocity integral varied from $1.5 \mathrm{~cm}$ at the annulus in subject 3
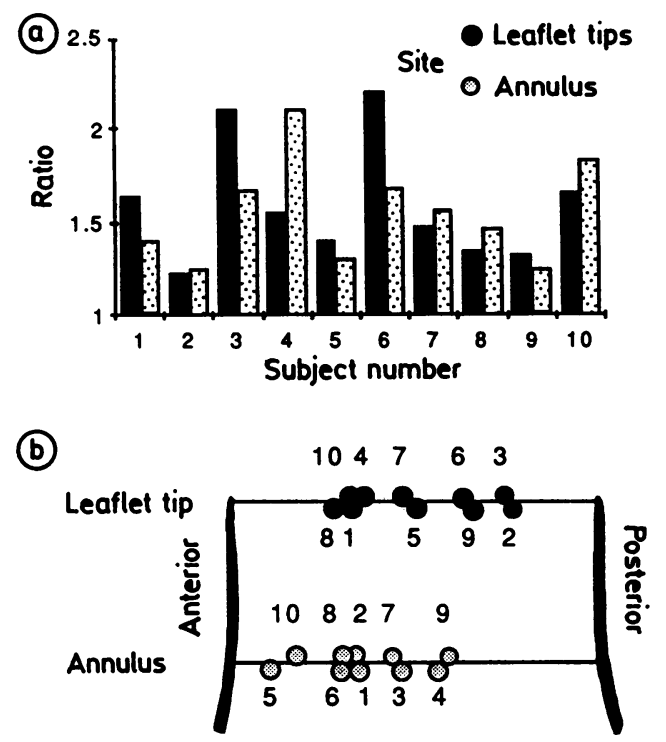

Fig 5 (a) Ratio of maximum time-velocity integral to the mean cross sectional time-velocity integral of early mitral flow at the leaflet tips (solid bars) and at the annulus (patterned bars). (b) Site of the maximum time-velocity integral within the mitral orifice is shown for each subject at the level of the tips (solid dots) and the annulus (open circles). Leaflet margins are indicated as solid vertical lines. up to $6 \cdot 1 \mathrm{~cm}$ at the leaflet tips in subject 6 (table). The mean differences for all subjects were $2.8 \mathrm{~cm}$ at the leaflet tips and $2.5 \mathrm{~cm}$ at the annulus. Figure 5 shows the ratio of the maximum integral to the mean time velocity integral, and the relative location of the maximum integral within the orifice for each man at the levels of the leaflet tips and the annulus.

Heart rate ranged from 41 to 76 (men $61(10)$ ) beats per minute (table). All men were in sinus rhythm.

\section{Discussion}

The results indicated that the velocity profiles, both at the tips of the mitral valve leaflets and at the annulus, are skewed to a variable degree during the early phase of the mitral flow. At the time of peak flow, the highest velocities were 1.2 to 2.2 (tip of the leaflets) and 1.2 to $2 \cdot 1$ (annulus) times higher than the cross sectional mean velocity. Similar results were found when the highest time-velocity integral was compared with the mean time-velocity integral across the orifice, indicating that the profiles were skewed to some extent throughout the early phase of the mitral flow. There were no clear differences in the degree of skewness at the tip of the leaflets and at the annulus. The average overestimation of the mean velocity integral that would occur if the maximal integral had been used was $49 \%$ at the tip of the leaflets and $46 \%$ at the annulus. The standard deviation, however, was larger at the tip of the leaflets (27\% v 15\%).

The orientation of the skew within the valve apparatus varied between individuals and in some men between the annulus and the leaflet tips (fig 4); it also varied with time (fig 3). This observation suggests that the recording of maximum flow 
velocity with pulsed wave Doppler ultrasound should not be guided by the location in the valve orifice at a defined depth, but by the observed maximum velocities only.

In one of the men (number 1, table) we found a higher maximum flow velocity at the mitral annulus than at the leaflet tips. This is in contrast with what is usually found with pulsed Doppler in patients and might possibly be explained by the suboptimal orientation of the plane of the sweep sector because both cross sectional profiles were obtained from the same sweeps. The small difference in maximum velocities between the annulus and the leaflet tips in four of the other men may similarly have been caused by a suboptimal anterioposterior orientation of the plane of the flow sector, but might also be explained by small differences in orifice size at the two locations.

Few attempts have been made to measure the velocity profiles of mitral flow because its location within the heart makes it difficult to measure. Invasive procedures may interfere with valvar or ventricular function and this may affect the mitral flow pattern.

We are aware of only one previous published study on the velocity profiles of mitral flow. ${ }^{9}$ Taylor and Whamond used a Pitot needle to study flow through the mitral orifice in dogs. They reported fairly flat profiles of early mitral flow both at the annulus and at the tip of the valve leaflets. Taylor's results and our own may differ because we studied different species and used different techniques.

Previous estimates of volume flow based on measurements of flow velocity and flow area at the level of the mitral valve assumed a flat velocity profile. ${ }^{1011}$ Our results suggest that values based on the maximum time velocity integral tend to overestimate the mean integral. The inconsistency of the results of different studies may partly be explained by our findings of a non-flat velocity profile and partly by inaccuracies in measurements of the effective flow area. ${ }^{12-15}$

\section{APPLICATIONS}

Colour flow Doppler provides a new way of evaluating velocity profiles within the heart because the velocity information from sample volumes located along an arc at a certain depth can be analysed. The advantage of this method is that the ultrasound beam can be oriented almost in parallel with the flow velocity. The resulting cross sectional velocity profiles will thus contain more accurate velocity information. However, the main problem is that these velocity profiles will be skewed when recordings are made of accelerating flow because of the time taken to scan the flow sector.
There are several reports on flow topography based on Doppler two dimensional flow recordings. ${ }^{46}$ One of them was an in vivo study in which the time lag of the colour Doppler technique may have introduced errors because of the changes in flow velocity during each flow scan. ${ }^{5}$ So far the timing of the colour flow sweep in relation to the events during the cardiac cycle has been discussed only in relation to regurgitation jets. ${ }^{16} 17$

The present method avoids the distortion of the velocity profile caused by the time lag, making it possible to obtain accurate velocity profiles at different sites within the heart. This has important implications for volume flow calculations, especially in regurgitation and with shunts.

\section{OTHER METHODS}

Multigated Doppler ultrasound has been used by some. ${ }^{18} 19$ But with this method the flow velocity has to be recorded nearly perpendicularly to the direction of the ultrasound beam to read the flow velocity profile across the width of a flow channel. This is a disadvantage because errors in the velocity estimate increase as a function of the cosine of the angle between the blood flow and the ultrasound beam.

Magnetic resonance techniques for velocity mapping have been used on the ascending and descending aorta in healthy individuals, ${ }^{2021}$ but so far flow velocity profiles across the mitral orifice have not been reported.

In experimental settings different methods have been used, including the Pitot principle and hot film anemometry, ${ }^{22}$ but the invasiveness of these methods limits their use in patients. Pulsed wave Doppler ultrasound, with the ultrasound beam directed in parallel with the blood flow direction, has been used to measure flow in the ascending aorta during operation. ${ }^{24}$

\section{LIMITATIONS OF THE METHOD}

The main limitation of the method used in the present study was that the profiles were evaluated in only one plane and from only one sequence of time gated recordings in each man. This may explain why the highest velocities were recorded at the annulus in some men. At the tip of the leaflets the highest velocities may have been outside the plane of the colour flow sector. This limitation can be overcome by stepwise rotation of the transducer to construct a series of profile maps. With current instruments and software such a procedure would be too time consuming.

Because the streamlines of the mitral flow probably ran in parallel, at least during part of diastole, and the ultrasound beams making up the Doppler colour 
sector did not, the angle of incidence between the flow and measurement direction varies from one side of the flow area to the other. To minimise the error on profile calculations we attempted to align the centreline of the Doppler sector with the centreline of the flow. A sector angle of $30^{\circ}$ was sufficient to cover the mitral flow in the region of interest, limiting the maximum angle of incidence to about $15 \%$. Accordingly, an error on velocity estimates should be less than $6 \%$. When the velocity pattern is more complicated (that is the streamlines are not parallel) or when the vector of the velocities is out of the plane of the flow velocity sector the error may be larger.

The velocity estimates at each point in the two dimensional flow map are based on calculations of the Doppler shift of the received signal from each point in the sector. In conventional Doppler recordings, fast Fourier transformation is used to give a spectrum display as well as maximum and mean estimates. Because the two dimensional flow technique has a much faster rate of data acquisition than conventional Doppler, more efficient processing of the data is required. The algorithm used by the instrument is based on calculation of the centre frequency (that is the mean frequency of the Doppler spectrum from each point in the flow sector) and intensity of the received signal based on an autocorrelation technique. If flow is not disturbed this method gives accurate velocity estimates. ${ }^{78}$

The lateral resolution of the system caused two neighbouring points in the three dimensional plots to overlap at a position where the power of the backscattered signals was reduced to $60 \%$ of the maximum power centrally in each beam (fig 3). The influence of points that were further apart was not significant. Hence the resolution of the three dimensional plots was regarded as appropriate.

The high pass filter of the instrument used was set at $21 \mathrm{~cm} / \mathrm{s}$, which means that velocities below this limit were not recorded. The upper velocity range is limited by the Nyquist frequency. ${ }^{25}$ However, by moving the baseline this limit can be increased up to two times the Nyquist velocity minus the high pass filter velocity limit. We used a $3 \mathrm{MHz}$ transducer with an upper velocity limit of $120 \mathrm{~cm} / \mathrm{s}$.

The two different routines for transferring the digital ultrasound data from flow sweeps introduced no differences in the calculation of flow velocities because both methods used the same algorithm to calculate velocity. Also we calculated the instantaneous flow velocity profiles in the same way in all the men-by linear time interpolation between the acquired data from the sequentially delayed flow maps.

\section{SOURCES OF ERRORS}

Because the recording technique samples data from several heart beats it requires exact aiming, without transducer movement during the recording period. Thoracic movement and changes in mitral flow volume caused by respiration were likely to introduce errors. So the men were asked to stop respiration in passive end expiration with open airways during data acquisition.

Changes in the selected depth of sampling caused by volume filling of the left ventricle during the recording period were not corrected because the change in location of the mitral annulus within the two dimensional flow sector was considered to be small during early diastole.

Irregularities in heart rate and rhythm, as well as the increment in the sequentially delayed two dimensional flow maps relative to the trigger marker (the $\mathbf{R}$ wave of the electrocardiogram signal), could also introduce errors in calculations of the instantaneous flow velocity profile.

To reduce these sources of error we checked all recordings for inaccuracies by replaying video recordings. Because the two dimensional tissue sector is updated immediately before the two dimensional flow map, changes in tissue and flow sectors relative to the heart from one heart beat to the other could be monitored.

\section{CONCLUSIONS}

The use of digital flow velocity information from two dimensional Doppler flow maps of the early mitral flow, using time interpolation of the acquired data from sequentially delayed flow maps, provides a new method for calculation of instantaneous flow velocity profiles.

The results from our study give new information on the distribution of mitral flow velocity across the width of the mitral valve at the levels of the leaflet tips and at the annulus in the human heart. This shows that the instantaneous flow velocity profiles are variably skewed at both sample depths in an apical four chamber view. A skewed profile of early mitral flow velocity should be borne in mind when recordings from a small area in the valve orifice are used for volume flow calculations; this skewness may result in significant errors.

This study was supported by grants from the Norwegian Council on Cardiovascular Diseases.

\section{References}

1 Matsuo H, Kitabatake A, Hayashi T, et al. Intracardiac flow dynamics with bi-directional ultrasonic pulsed Doppler technique. Jpn Circ J 1977;41:515-28.

2 Kitabatake A, Michitoshi I, Asao M, et al. Transmitral blood flow reflecting diastolic behavior of the left 
ventricle in health and disease-a study by pulsed Doppler technique. Jpn Circ J 1982;46:92-102.

3 Appleton CP, Hatle LK, Popp RL. Relation of transmitral flow velocity patterns to left ventricular diastolic function: new insights from a combined hemodynamic and Doppler echocardiographic study. J Am Coll Cardiol 1988;12:426-40.

4 Miyatake K, Izumi S, Shimizu A, et al. Right atrial flow topography in healthy subjects studied with real-time two-dimensional Doppler flow imaging technique. J Am Coll Cardiol 1986;7:425-31.

5 Wollenweber A, Redel DA. Is there a flat velocity profile in the left ventricular outflow tract? Heart and Vessel 1987;suppl 3:49.

6 Sahn DJ, Tamura T. Development of a method for computer processing of flow map images. Heart and Vessel 1987;suppl 3:22.

7 Kasai C, Namekawa K, Koyano A, Omoto R. Real-time two-dimensional blood flow imaging using an autocorrelation technique. IEEE Transactions on Sonics and Ultrasonics 1985;SU-32:458-64.

8 Kristoffersen $\mathrm{K}$. Time domain estimation of the center frequency and spread of Doppler spectra in diagnostic ultrasound. IEEE Transactions on UFFC 1988;35:685-700.

9 Taylor DEM, Whamond JS. Velocity profile and impedance of the healthy mitral valve. In: Kalmanson D, ed. The mitral valve. A pluridisciplinary approach. Acton, Massachusetts: Publishing Sciences Group, 1976:127.

10 Zhang Y, Nitter-Hauge S, Ihlen H, Myhre E. Doppler echocardiographic measurement of cardiac output using the mitral orifice method. $\mathrm{Br}$ Heart $J$ 1985;53:130-36.

11 de Zuttere $D$, Touche $T$, Saumon G, Nitenberg A, Prasquier R. Doppler echocardiographic measurement of mitral flow volume: validation of a new method in adult patients. J Am Coll Cardiol 1988; 11:343-50.

12 Stewart WJ, Jiang L, Mich R, Pandian N, Guerrero JL, Weyman AE. Variable effects of changes in flow rate through the aortic, pulmonary and mitral valves on valve area and flow velocity: impact on quantitative Doppler flow calculations. J Am Coll Cardiol 1985;6:653-62.

13 Dittmann H, Voelker W, Karsch K-R, Seipel L.
Influence of sampling site and flow area on cardiac output measurements by Doppler echocardiography. J Am Coll Cardiol 1987;10:818-23.

14 Nicolosi GL, Pungercic E, Cervesato E, et al. Feasibility and variability of six methods for the echocardiographic and Doppler determination of cardiac output. Br Heart J 1988;59:299-303.

15 Robson SC, Boys RJ, Hunter S. Doppler echocardiographic estimation of cardiac output: analysis of temporal variability. Eur Heart J 1988;9:313-8.

16 Kyo S, Kondo Y, Takamoto S, Matsumura M, Yokote Y, Omoto R. Scanning frame rate and direction dependent variability in flow image of phased-array color Doppler: computer-graphic analysis. Circulation 1987;76 (suppl 4):4-526.

17 Smith MD, Grayburn PA, Spain MG, DeMaria AN, $\mathrm{K}$ wan OL, Moffett CB. Observer variability in the quantitation of Doppler color flow jet areas for mitral and aortic regurgitation. J Am Coll Cardiol 1988; 11:579-84.

18 Jenni R, Vieli A, Ruffimann K, Krayenbuehl HP, Anliker M. A comparison between single gate and multigate ultrasonic Doppler measurements for the assessment of the velocity pattern in the human ascending aorta. Eur Heart J 1984;5:948-53.

19 Jenni R, Ruffmann K, Vieli A, Anliker M, Krayenbuehl HP. Dynamics of aortic flow in hypertrophic cardiomyopathy. Eur Heart J 1985;6:391-98.

20 Nayler GL, Firmin DN, Longmore DB. Blood flow imaging by cine magnetic resonance. J Comput Assist Tomogr 1986;10:715-22.

21 Klipstein RH, Firmin DN, Underwood SR, Rees RSO, Longmore DB. Blood flow patterns in the human aorta studied by magnetic resonance. Br Heart $J$ 1987;58:316-23.

22 Seed WA, Wood NB. Velocity patterns in the aorta. Cardiovasc Res 1971;5:319-30.

23 Paulsen PK, Hasenkam JM. Three-dimensional visualization of velocity profiles in the ascending aorta in dogs, measured with a hot-film anemometer. $J$ Biomech 1983;16:201-10.

24 Segadal L, Matre K. Blood velocity distribution in the human ascending aorta. Circulation 1987;76:90-100.

25 Hatle L, Angelsen B. Doppler ultrasound in cardiology. Physical principles and clinical applications. Philadelphia: Lea and Febiger, 1985:63. 